\title{
Small-angle X-ray and neutron scattering analyses of highly crosslinked rubber with unsaturated carboxylic acid
}

\author{
Ryo Mashita ${ }^{1}$, Hiroyuki Kishimoto ${ }^{1}$, Rintaro Inoue ${ }^{2}$ and Toshiji Kanaya ${ }^{2}$
}

We studied the structure of polybutadiene rubber (BR) crosslinked with zinc diacrylate (ZDA) by using small-angle X-ray scattering (SAXS) and small-angle neutron scattering (SANS) to elucidate the regions of high crosslink density in the matrix rubber. The SAXS profiles of the swollen rubber materials with toluene were described well by a model that considers the hierarchical structure of the ZDA aggregates. By contrast, the SANS profiles of the swollen rubber materials with deuterated toluene were not well described by the same model, suggesting an influence of the high crosslink density region in the matrix rubber. We then considered the contributions of the high crosslink density segments of the BR (HC-BR) and the hierarchical structure of the ZDA aggregates, and succeeded in describing the SANS curves fairly well. This paper is the first report on high crosslink density segments in BR crosslinked with ZDA. We also performed tensile measurements on the sample and compared the results with those from the SAXS/SANS measurements, revealing that the size and the number density of the HC-BR and ZDA aggregates are related to the mechanical properties of the rubber material.

Polymer Journal (2013) 45, 57-63; doi:10.1038/pj.2012.200; published online 21 November 2012

Keywords: crosslink; polybutadiene; small-angle neutron scattering; small-angle X-ray scattering; zinc diacrylic acid

\section{INTRODUCTION}

At present, many industries, including the automobile industry, require lightweight materials with high tensile strengths and high abrasion resistances to improve the manufacturing of commercial products. Hence, controlling the morphology and structure of functional polymeric materials is indispensable to the development of next-generation materials.

The structure of rubber crosslinked with metal acrylate has been extensively studied because such rubber exhibits strong reinforcement properties without requiring the addition of reinforcing materials such as carbon black, silica or clay. Electron microscopy and scanning probe microscopy measurements of these rubber materials have revealed that metal ions formed aggregates with sizes of $10-30 \mathrm{~nm},{ }^{1-4}$ and these aggregates have been presumed to be responsible for these materials' mechanical properties. Therefore, many studies have focused on the metal ion aggregates in these rubber materials ${ }^{5-9}$ with the goal of improving the materials' mechanical properties. However, no dramatic improvement in the mechanical properties has been reported. By contrast, the structure of the matrix rubber, which is a primary component in these rubber materials, has not been studied in detail because of its complexity. Elucidating the structure of the matrix rubber could provide a promising approach to enhance the mechanical properties of these rubber materials, and we thus decided to analyze the structure of the matrix rubber. Transverse proton $\left({ }^{1} \mathrm{H}\right)$ NMR relaxation measurements have revealed a strong correlation between the crosslink density in the matrix rubber and the residual dipolar coupling, and this method was therefore applied to characterize the crosslink density of the rubber materials. ${ }^{10-12}$ Two relaxation processes were observed in the matrix rubber, ${ }^{13}$ implying the coexistence of high crosslink density and low crosslink density regions in the matrix rubber. Transmission electron microscopy (TEM) and scanning electron microscopy (SEM) measurements were performed on the matrix rubber to confirm the existence of two regions with different crosslink densities. However, the coexistence of two regions in the matrix rubber was not clarified because of a lack of contrast in the TEM and SEM measurements, which is determined by the difference in the electron densities.

If regions with high crosslink density and low crosslink density coexist in the matrix rubber, the high crosslink density region would swell less than the low crosslink density region when the matrix rubber is immersed in a good solvent. This method must be used to enhance the contrast difference between the high and low crosslink density regions. How can we detect the difference between the regions? In the case of neutron scattering, the contrast is provided by the scattering length density: for example, hydrogen atoms $(\mathrm{H})$ are distinct from deuterium (D) atoms because of their large difference in scattering length. If the (hydrogenated) matrix rubber is swollen in 
deuterated solvent, it would be possible to distinguish high crosslink density rubber from low crosslink density rubber (LC-BR) by using small-angle neutron scattering (SANS) measurements. In fact, SANS studies on swollen rubber have successfully revealed the complex structure of rubber materials, ${ }^{14-16}$ and thus SANS is a promising technique for unveiling the hidden structure of the high crosslink density region in the rubber material.

For our rubber material, it is also necessary to evaluate the structure and spatial distribution of the metal acrylate to reliably analyze the matrix rubber structure, that is, we must analyze the structures of both the metal acrylate and the matrix rubber, including high and low crosslink density regions, without ambiguity. Complementary use of small-angle X-ray scattering (SAXS) and SANS is suited for this purpose. Specifically, the structure of metal acrylate is analyzed using SAXS measurements, and then the structure of the matrix rubber can be analyzed using SANS measurements and the results of the SAXS measurements.

In this study, we investigated the structure of butadiene rubber crosslinked with metal acrylate and primarily focused on determining whether regions of high crosslink density and low crosslink density coexist in the matrix rubber by using SAXS and SANS measurements. By comparing the SAXS and SANS results with the tensile moduli, we also clarified the correlation between the structure and the mechanical properties of the studied rubber materials.

\section{EXPERIMENTAL PROCEDURE}

\section{Materials and sample preparation}

The details of the materials used in the present study are summarized in Table 1. We used polybutadiene rubber (BR; BR730, JSR Co., Ltd., Tokyo, Japan) as the matrix rubber. Zinc diacrylate (ZDA; Sanceler synchrotron radiation, Sanshin Chemical Industry Co., Ltd., Yanai, Yamaguchi, Japan) and dicumyl peroxide (Percmyl D, Nichiyu Co., Ltd., Tokyo, Japan) were used as a crosslinking agent and a polymerization initiator of butadiene, respectively. The components were mixed using a 6-inch two-roll mill and molded into sheets $1 \mathrm{~mm}$ thick at $170^{\circ} \mathrm{C}$ for $20 \mathrm{~min}$ (Sample A) and $230^{\circ} \mathrm{C}$ for $5 \mathrm{~min}$ (Sample B). Before the SAXS and SANS measurements, Samples A and B were swollen in toluene and deuterated toluene, respectively, and reached their equilibrium states at room temperature (final swollen ratio was $\sim 300 \%$ ). The swollen samples were measured in an optical cell $2 \mathrm{~mm}$ thick.

\section{Measurements}

Rubber materials exhibit hierarchical structures over a wide range of spatial scales, and the structures at different spatial scales are intricately correlated to each other. Hence, investigating the structure of rubber materials over a wide range of spatial scales is indispensable for clarifying the correlations between a rubber material's structure and its mechanical properties. Hence, we studied the structure of rubber over a wide $q$ range using SAXS and SANS, where $q$ is the magnitude of the scattering vector and is defined by

$$
q=\frac{4 \pi \sin (\theta)}{\lambda}
$$

Table 1 Details about the components of the samples used in the present study

\begin{tabular}{lcc}
\hline & Sample A & Sample B \\
\hline Polybutadiene (vol\%) & 87.2 & 87.2 \\
Zinc diacrylate (vol\%) & 11.6 & 11.6 \\
Dicumyl peroxide (vol\%) & 1.1 & 1.1 \\
Crosslinking temperature $\left({ }^{\circ} \mathrm{C}\right)$ & 170 & 230 \\
Crosslinking time (min) & 20 & 5 \\
\hline
\end{tabular}

where $2 \theta$ and $\lambda$ are the scattering angle and wavelength of the incident beam, respectively.

SAXS measurements were performed using BL03XU and BL40B2 instruments installed at a synchrotron radiation X-ray scattering facility, SPring-8, in Nishiharima, Japan. The incident wavelength of the X-ray and the sample-todetector distance were $1.5 \AA$ and $3.0 \mathrm{~m}$, respectively. Ultra small-angle X-ray scattering (USAXS) measurements were also performed using a BL19B2 instrument installed at SPring-8, and the incident wavelength of the X-ray and the sample-to-detector distance were $0.69 \AA$ and $41 \mathrm{~m}$, respectively. By combining the SAXS and USAXS measurements, we can cover a $q$ range from $4.6 \times 10^{-4}$ to $8.7 \times 10^{-2} \AA^{-1}$. The two-dimensional scattering images were circularly averaged to obtain one-dimensional scattering curves, which were corrected for the backgrounds from the sample cell and the air, and for the transmittance of the X-rays through the samples.

SANS measurements were performed using a SANS-J-II spectrometer installed at the Japan Research Reactor- 3 in Tokai, Japan. The wavelength $\lambda$ of the incident neutron and its distribution $(\Delta \lambda / \lambda)$ were $6.56 \AA$ and $0.11 \AA$, respectively. The SANS measurements were performed using sample-todetector distances of $2.5 \mathrm{~m}$ and $10 \mathrm{~m}$, and we also used a focusing lens to obtain a lower $q$ region than that covered by the conventional set-up. Using this set-up, the $q$ values ranged from $8.5 \times 10^{-4}$ to $1.2 \times 10^{-1} \AA^{-1}$. The observed two-dimensional scattering images were circularly averaged to obtain one-dimensional scattering curves, which were corrected for the backgrounds from the sample cell and the air, the electronic noise, the detector sensitivity, the transmittance of the neutrons through the samples and incoherent scattering. The SANS scattering intensity $(I(q))$ was converted to an absolute intensity scale $\left(\mathrm{cm}^{-1}\right)$ using a porous aluminum plate as a secondary standard. ${ }^{17}$

SEM and TEM measurements were performed at operating voltages of $15 \mathrm{kV}$ and $200 \mathrm{kV}$, respectively. Tensile measurements were performed using a tensile tester with a cross-head speed of $200 \mathrm{~mm} \mathrm{~min}^{-1}$ at room temperature.

\section{RESULTS AND DISCUSSION}

A large number of micrometer-scale particles were observed in both Samples A and B in the SEM measurements, as shown in Figure 1. The ZDA monomer has a diameter of $\sim 1.4 \mathrm{~nm}$, so the particles observed through SEM were ZDA aggregates. Image analysis reveals that the average diameters of the ZDA aggregates in Samples A and B were $1.8 \mu \mathrm{m}$ and $3.3 \mu \mathrm{m}$, respectively. To observe the morphology on a smaller spatial scale, we also performed TEM measurements on the samples. The matrix BR areas exhibiting no ZDA aggregates on the micrometer scale were selected for the TEM measurements. Two types of ZDA aggregates with diameters of $\sim 5 \mathrm{~nm}$ (first ZDA aggregates) and $\sim 20 \mathrm{~nm}$ (second ZDA aggregates) were observed in the TEM images, as shown in Figure 2. Although it is difficult to accurately analyze the TEM and SEM images because of their limited spatial resolutions, it is safe to say that the ZDA aggregates exhibit a wide distribution of sizes or a hierarchical structure over a wide range of spatial scales in both Samples A and B. The number of ZDA aggregates seems to be larger in Sample A than in Sample B. The difference in ZDA aggregation in terms of size and number might be related to differences in the mechanical properties of Samples A and $\mathrm{B}$, which will be discussed later.

The scattering length density and the electron density of each component in the samples and solvents (toluene and deuterated toluene) are listed in Table 2. In the SAXS measurements, it is assumed that the scattering intensity from ZDA dominates the total scattering intensity because the difference in the electron densities of ZDA and toluene is much larger than the difference between the electron densities of the matrix BR and toluene. If some regions of the matrix BR have higher crosslink densities than the rest, these higher crosslink density regions will not swell in deuterated toluene as much as the lower density regions, resulting in a difference in the scattering 
length densities. As a result, the difference in the crosslink density will be observable in the SANS profile through the difference in the scattering length density between the high crosslink density region and the deuterated toluene. To verify the assumptions mentioned above, we focus on the experimentally obtained SAXS and SANS scattering profiles.

The intensity profiles $I(q)$ of SAXS $\left(I_{\text {SAXS }}(q)\right)$ and SANS $\left(I_{\text {SANS }}(q)\right)$ from Sample A are shown in Figure 3a and those from Sample B are shown Figure $3 \mathrm{~b}$. The $I_{\mathrm{SAXS}}(q)$ 's were shifted vertically to overlap with the $I_{\text {SANS }}(q)$ 's in the $q$ range of $q<0.001 \AA^{-1}$ and $q>0.05 \AA^{-1}$ for comparison. The difference in $I_{\mathrm{SAXS}}(q)$ and $I_{\mathrm{SANS}}(q)$ is especially observable over the $q$ region ranging from 0.002 to $0.02 \AA^{-1}$. As we have already explained above, the difference in the SAXS and SANS profiles originates primarily from the coexistence of high and low crosslink density regions in the matrix rubber.

A model function that considers the hierarchical structure of ZDA aggregates, which were determined from the TEM and SEM images (Figures 1 and 2), is used to describe the $I_{\mathrm{SAXS}}(q)$ scattering profiles. The $I(q)$ of rubber in which silica particles are dispersed hierarchically
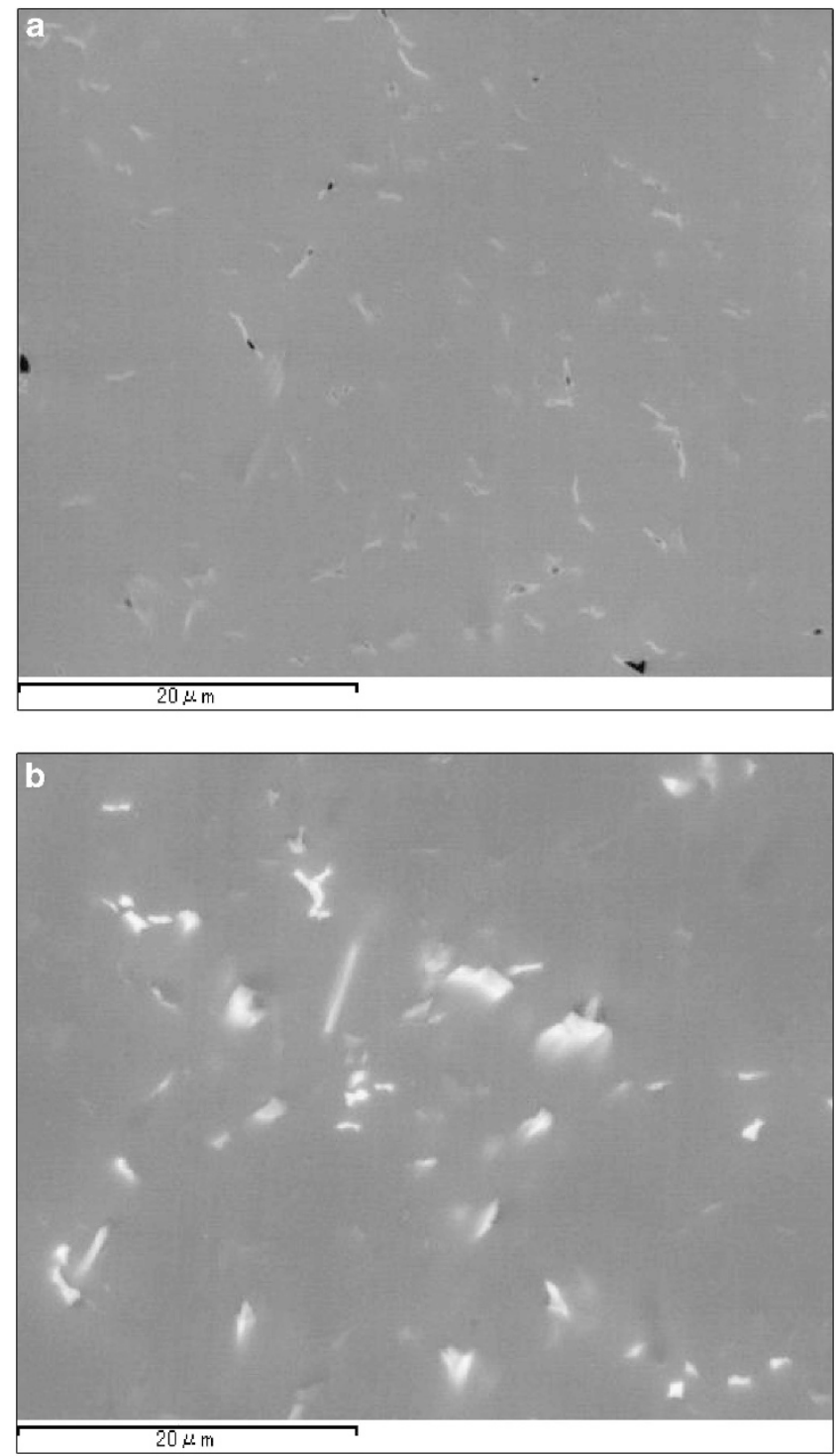

Figure 1 SEM images of (a) Sample A and (b) Sample B. has been successfully analyzed using a unified approach, ${ }^{18-21}$ and we also applied the model given by Equation 2 as a trial.

$$
\begin{aligned}
& I(q)=P_{4}\left[\left\{\operatorname{erf}\left(\frac{q R_{\mathrm{g}, 4}}{\sqrt{6}}\right)^{3} / q\right\}\right]^{F_{4}} \exp \left(\frac{-q^{2} R_{\mathrm{g}, 4}^{2}}{3}\right)+G_{3} \exp \left(\frac{-q^{2} R_{\mathrm{g}, \mathrm{BR}}^{2}}{3}\right) \\
& +P_{3}\left[\left\{\operatorname{erf}\left(\frac{q R_{\mathrm{g}, \mathrm{BR}}}{\sqrt{6}}\right)^{3} / q\right\}\right]^{F_{\mathrm{BR}}}+G_{2} \exp \left(\frac{-q^{2} R_{\mathrm{g}, \mathrm{ZDA} 2}^{2}}{3}\right) \\
& +P_{2}\left[\left\{\operatorname{erf}\left(\frac{q R_{\mathrm{g}, \mathrm{ZDA} 2}}{\sqrt{6}}\right)^{3} / q\right\}\right]^{F_{2}} \exp \left(\frac{-q^{2} R_{\mathrm{g}, \mathrm{ZDA} 1}^{2}}{3}\right) \\
& +G_{1} \exp \left(\frac{-q^{2} R_{\mathrm{g}, \mathrm{ZDA} 1}^{2}}{3}\right)+P_{1}\left[\left\{\operatorname{erf}\left(\frac{q R_{\mathrm{g}, \mathrm{ZDA} 1}}{\sqrt{6}}\right)^{3} / q\right\}\right]^{F_{1}} \\
& \operatorname{erf}(x)=\frac{2}{\sqrt{\pi}} \int_{0}^{x} e^{-t^{2} \mathrm{~d} t}
\end{aligned}
$$

where $R_{\mathrm{g}, \mathrm{ZDA} 1}, R_{\mathrm{g}, \mathrm{ZDA} 2}, R_{\mathrm{g}, \mathrm{BR}}$ and $R_{\mathrm{g}, 4}$ are the radii of gyration of the first ZDA aggregates, the second ZDA aggregates, the high crosslink density BR (HC-BR) and the larger ZDA aggregates, respectively, and $R_{\mathrm{g}}$ is defined as the root mean square distance of the object's points from its center of gravity. $\operatorname{erf}(x)$ in Equation 2 corresponds to the error function, which is also known as the Gaussian error function and is defined in Equation 3. We did not consider cross-terms between the ZDA aggregates and the HC-BR or between ZDA aggregates of different size in Equation 2. We tentatively used values of $1.8 \mu \mathrm{m}$ and $3.3 \mu \mathrm{m}$ for $R_{\mathrm{g}, 4}$, which were determined from the SEM images of Samples A and B, respectively. The size of $R_{\mathrm{g}, 4}$ is out of the accessible $q$ range in the present USAXS and SAXS measurements. $F_{1}$, $F_{2}, F_{\mathrm{BR}}$ and $F_{4}$ are, the mass-fractal dimensions of the first ZDA aggregates, the second ZDA aggregates, the $\mathrm{HC}-\mathrm{BR}$ and the larger ZDA aggregates, respectively. $G_{1}, G_{2}$, and $G_{3}$ are the exponential prefactors and $P_{1}, P_{2}, P_{3}$ and $P_{4}$ are the power law prefactors. ${ }^{19-21}$

In practice, we have to include the scattering contributions from the LC-BR in Equation 2. The swelling ratio of the present rubber materials (Samples A and B) in deuterated toluene was estimated to be $\sim 300 \%$, suggesting a large fraction of LC-BR in the matrix rubber. The scattering contrast of SANS is generally given by the square of the difference between the average scattering length density and that of the component. If we assume that the average scattering length density of the matrix rubber is almost equal to that of LC-BR, then the scattering intensity of the matrix rubber is dominated by that of the HC-BR. We ignore the scattering contribution from the LC-BR in Equation 2 in a first trial.

Figures $4 \mathrm{a}$ and $\mathrm{b}$ show the results of fits of the $I_{\text {SAXS }}(q)$ distributions of Samples A and B, respectively, to Equation 2. The observed and calculated $I_{\text {SAXS }}(q)$ agree fairly well, indicating that the unified approach can describe the obtained scattering curves. Hence, we decided to apply the unified approach in further analysis of the SAXS and SANS scattering curves. We found that $G_{3}$ was nearly equal to zero $\left(G_{3} \simeq 0\right)$, implying that the scattering contribution from HC-BR is negligible in the SAXS profiles. This finding supports our initial assumption that the total SAXS intensity is dominated by the scattering from the ZDA aggregates. The fit values of $R_{\mathrm{g}, \mathrm{ZDA} 1}$ and $R_{\mathrm{g}, \mathrm{ZDA} 2}$ are $5.0 \mathrm{~nm}$ and $14.0 \mathrm{~nm}$ for Sample A, respectively, and $4.8 \mathrm{~nm}$ and $19.4 \mathrm{~nm}$ for Sample B, respectively.

For the curve fits to the SANS profiles, we used the values of $R_{\mathrm{g}, \mathrm{ZDA} 1}$ and $R_{\mathrm{g}, Z \mathrm{DA} 2}$ determined from SAXS analysis and $R_{\mathrm{g}, 4}$ from the SEM images. As noted above, we were able to observe systematic deviations in the scattering curves of the SANS and SAXS profiles in the $q$ range from 

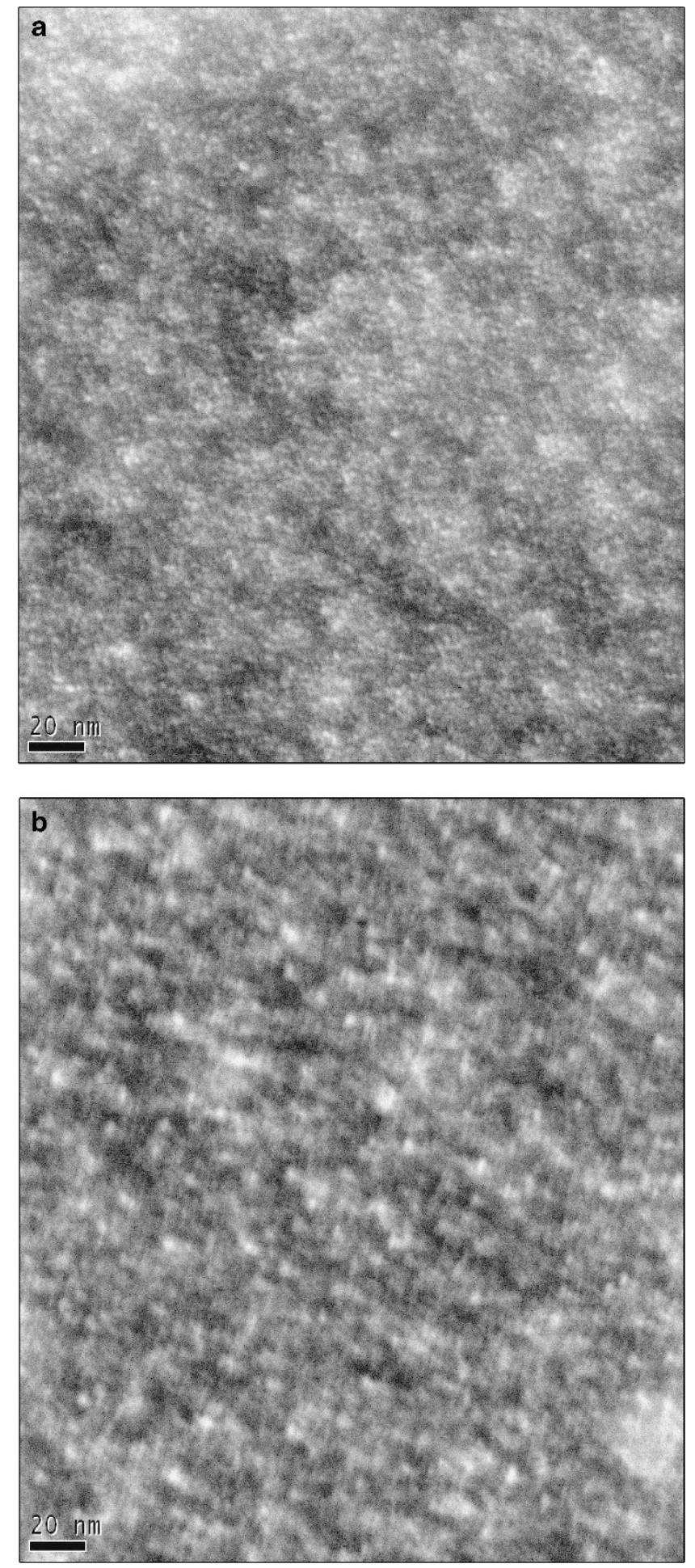

Figure 2 TEM images of (a) Sample A and (b) Sample B.

0.002 to $0.02 \AA^{-1}$, and these deviations surely indicate the scattering contribution of HC-BR in the SANS profiles. We would like to demonstrate that the $\mathrm{HC}-\mathrm{BR}$ component is necessary to quantitatively reproduce the SANS profiles, and so we tried to fit the unified approach function (Equation 2) to the SANS profiles without including the HCBR contribution $\left(G_{3}=0\right)$. The best fits to $I_{\text {SANS }}(q)$ under the constraint that $G_{3}=0$ are shown in Figures $5 \mathrm{a}$ and $\mathrm{b}$, and severe deviations were
Table 2 Scattering length densities and electron densities of the materials used in the present study

\begin{tabular}{lcc}
\hline & $\begin{array}{c}\text { Scattering length den- } \\
\text { sity }\left(\times 10^{-6} \AA^{-2}\right)\end{array}$ & $\begin{array}{c}\text { Electron density } \\
\left(\times 10^{23} \text { electron }^{-3}\right)\end{array}$ \\
\hline Polybutadiene & 0.42 & 3.01 \\
Zinc diacrylate & 2.15 & 4.83 \\
Toluene & 0.94 & 2.83 \\
Deuterated toluene & 5.16 & 2.82 \\
\hline
\end{tabular}
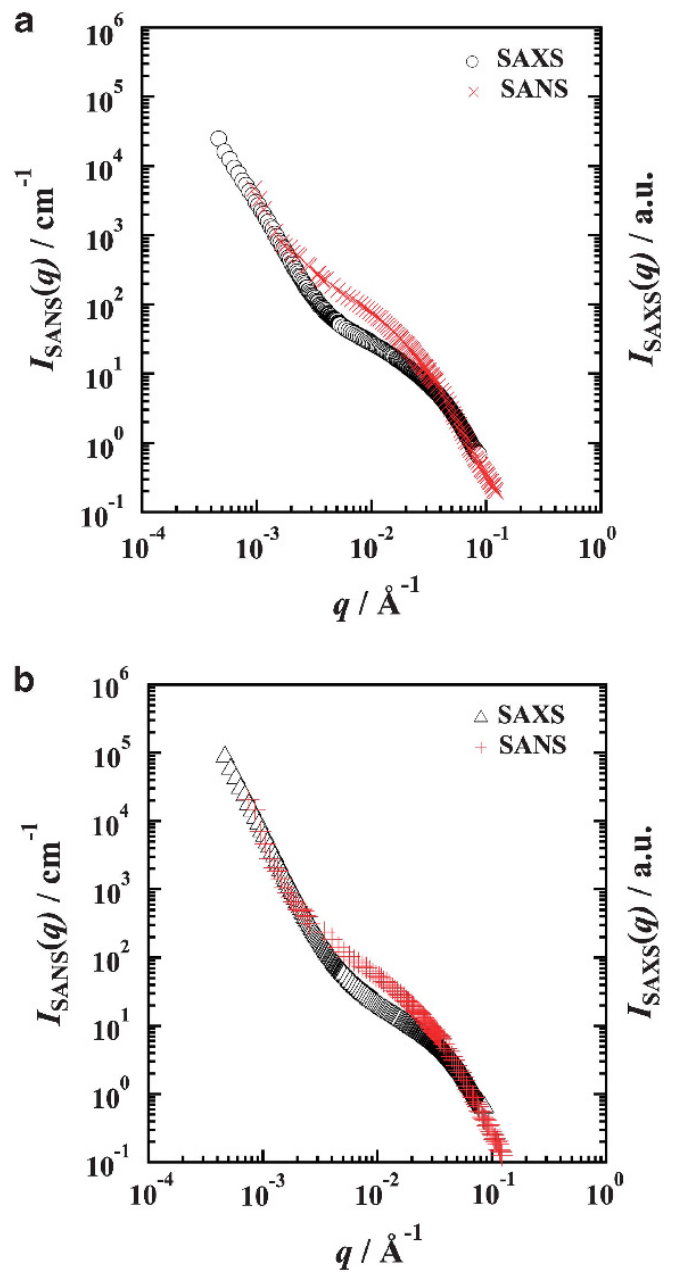

Figure 3 SAXS and SANS profiles of (a) Sample A and (b) Sample B.

observed over the $q$ range $2 \times 10^{-3}-0.01 \AA^{-1}$, implying that the HC-BR contribution is necessary to quantitatively reproduce the SANS profiles. We then fitted the model function (Equation 2) to $I_{\text {SANS }}(q)$ including the contribution of HC-BR $\left(G_{3} \neq 0\right)$ and found that $I_{\text {SANS }}(q)$ was well fitted to Equation 2 over the entire studied $q$ range for both Samples A and B, as shown in Figures $5 \mathrm{a}$ and b, respectively. As stated above, we neglected the scattering contribution from the LC-BR in the model 

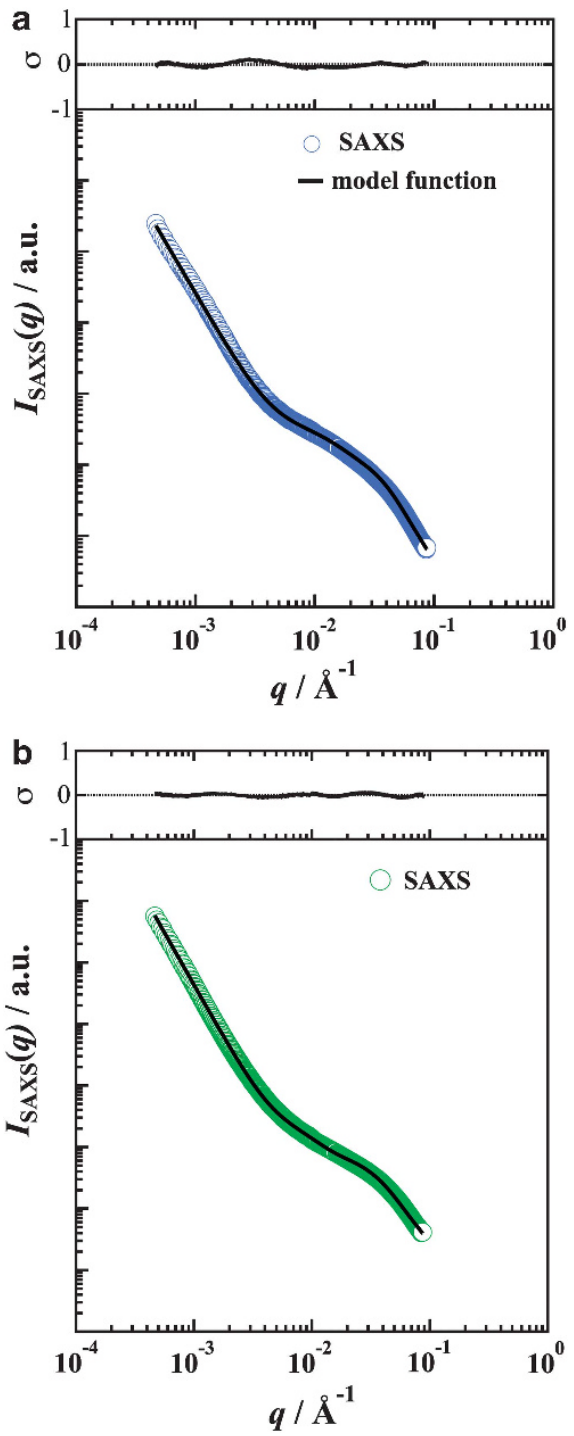

Figure 4 SAXS profiles of (a) Sample A and (b) Sample B. The solid lines are the results of fits to the model function, and $\sigma$ corresponds to the residuals.

function because of the small contrast between the swollen rubber and the deuterated toluene. The results of the fits to the SANS profiles without this contribution are quite satisfactory, supporting our initial assumption regarding the LC-BR.

In addition to evaluating the size of the HC-BR, the SANS analysis also determines the number densities $(N)$ of the ZDA aggregates, and the HC-BR from $G_{1}, G_{2}$ and $G_{3}$ because $I_{\text {SANS }}(q)$ was measured on an absolute scale. $G_{1}, G_{2}$ and $G_{3}$ are related to the number densities $(N)$ through the following equations.

$$
\begin{aligned}
G_{1} & =N_{\mathrm{ZDA} 1} V_{\mathrm{ZDA} 1}^{2} \Delta \rho_{\mathrm{ZDA}}^{2} \\
G_{2} & =N_{\mathrm{ZDA} 2} V_{\mathrm{ZDA} 2}^{2} \Delta \rho_{\mathrm{ZDA}}^{2} \\
G_{3} & =N_{\mathrm{BR}} V_{\mathrm{BR}}^{2} \Delta \rho_{\mathrm{BR}}^{2}
\end{aligned}
$$

$N_{\mathrm{ZDA} 1}, N_{\mathrm{ZDA} 2}$ and $N_{\mathrm{BR}}$ are the number densities of the first ZDA aggregates, the second $\mathrm{ZDA}$ aggregates and the $\mathrm{HC}-\mathrm{BR}$, respectively.
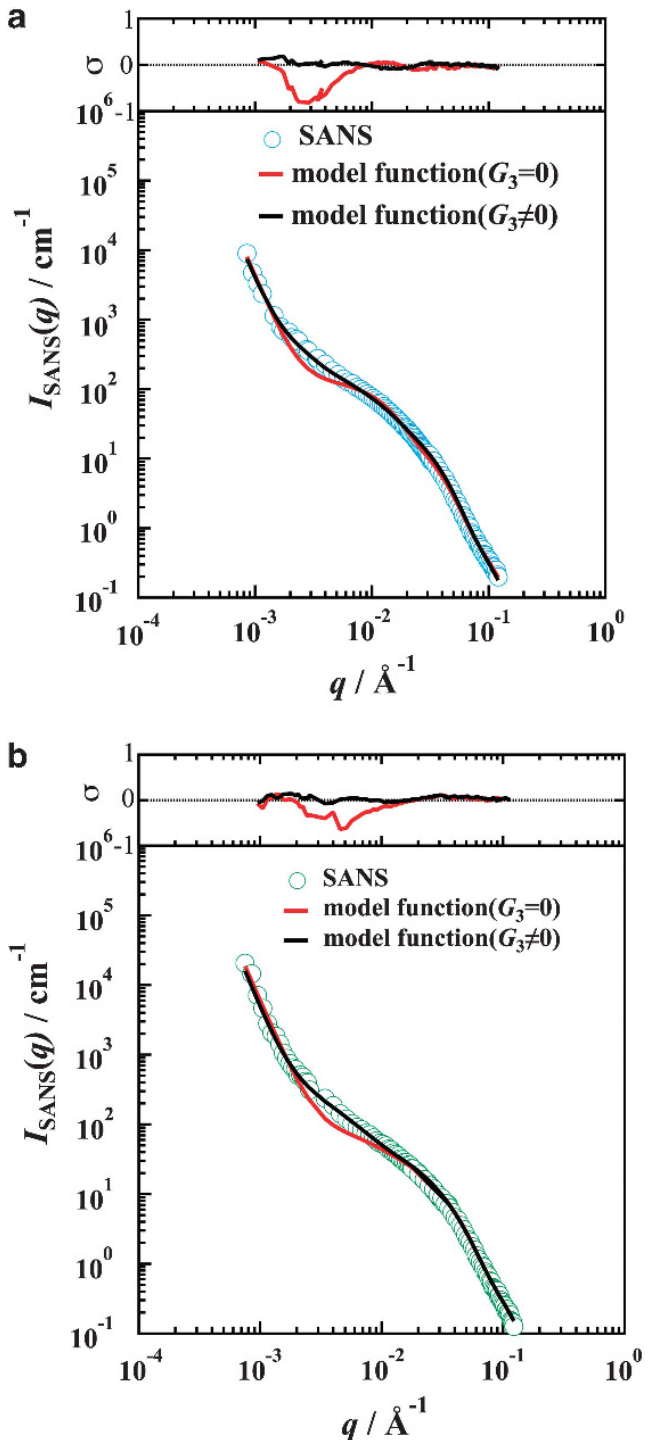

Figure 5 SANS profiles of (a) Sample A and (b) Sample B. The red solid lines and black solid lines are the results of fits to the model function without the HC-BR component $\left(G_{3}=0\right)$ and that with the HC-BR component, respectively. $\sigma$ Corresponds to the residuals.

$V_{\mathrm{ZDA} 1}, V_{\mathrm{ZDA} 2}$ and $V_{\mathrm{BR}}$ are the volumes of the first ZDA aggregates, the second $\mathrm{ZDA}$ aggregates and the HC-BR per unit volume, respectively. $\Delta \rho_{\mathrm{ZDA}}, \Delta \rho_{\mathrm{BR}}$ are the differences between the scattering length densities of ZDA and the average scattering length density and between $\mathrm{BR}$ and the average, respectively. The average scattering length density is evaluated considering the components of the rubber material summarized in Table 2. We have evaluated the number densities of the ZDA aggregates based on the results of these fits. We could not explicitly evaluate the number density of HC-BR because of a lack of knowledge about the degree of swelling of HC-BR. Hence, we only determined the minimum number density of HC-BR $\left(N_{\mathrm{BR}, \min }\right)$ as a reference by assuming that the scattering length density of HC-BR was the same as that of BR. The evaluated number densities of the ZDA aggregates, $N_{\mathrm{BR}}$, min , the $R_{\mathrm{g}}$ 's, the power law exponents and other evaluated parameters are summarized in Table 3 for both Samples A and B. $R_{\mathrm{g}, \mathrm{ZDA} 1}$ and $N_{\mathrm{ZDA} 1}$ were identical between Samples $\mathrm{A}$ and $\mathrm{B}$ independent of the crosslinking temperature 
Table 3 Radii of gyration $\left(\boldsymbol{R}_{\mathrm{g}}\right)$, number densities $(\boldsymbol{N})$, power law exponents of each component and other parameters obtained from the fits for Samples A and B

\begin{tabular}{lcc}
\hline & Sample $A$ & Sample B \\
\hline$R_{\mathrm{g}, \mathrm{ZDA} 1}(\mathrm{~nm})$ & 5.0 & 4.8 \\
$R_{\mathrm{g}, \mathrm{ZDA} 2}(\mathrm{~nm})$ & 14.0 & 19.4 \\
$R_{\mathrm{g}, \mathrm{BR}}(\mathrm{nm})$ & 72.2 & 109.2 \\
$N_{\mathrm{ZDA1}}\left(\mathrm{cm}^{3}\right)$ & $7.5 \times 10^{16}$ & $6.1 \times 10^{16}$ \\
$N_{\mathrm{ZDA2}}\left(\mathrm{cm}^{3}\right)$ & $1.1 \times 10^{15}$ & $1.3 \times 10^{14}$ \\
$N_{\mathrm{BR}, \min }\left(\mathrm{cm}^{3}\right)$ & $3.0 \times 10^{11}$ & $7.0 \times 10^{10}$ \\
$P_{1}$ & $2.4 \times 10^{-4}$ & $2.0 \times 10^{-4}$ \\
$P_{2}$ & $1.5 \times 10^{-3}$ & $1.6 \times 10^{-2}$ \\
$P_{3}$ & $1.4 \times 10^{-1}$ & $5.6 \times 10^{-3}$ \\
$P_{4}$ & $8.1 \times 10^{-9}$ & $6.8 \times 10^{-9}$ \\
$G_{1}$ & 17.3 & 11.7 \\
$G_{2}$ & 79.9 & 74.4 \\
$G_{3}$ & $6.2 \times 10^{2}$ & $1.3 \times 10^{3}$ \\
$F_{1}$ & 3.1 & 3.2 \\
$F_{2}$ & 1.8 & 1.8 \\
$F_{\mathrm{BR}}$ & 1.8 & 1.8 \\
$F_{4}$ & 3.9 & 4.0 \\
\hline
\end{tabular}

The minimum number density (NBR, min) of HC-BR was determined instead of the exact number density.

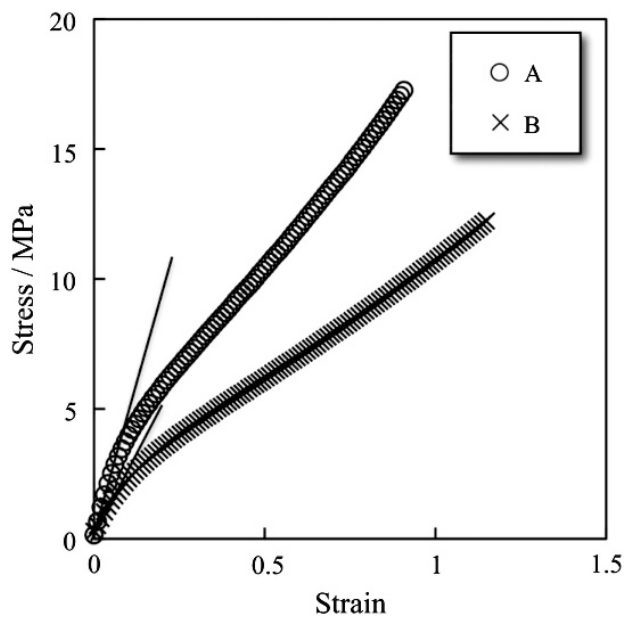

Figure 6 Strain dependence of stress in Samples A and B. The solid lines were used to calculate the elastic moduli.

(Table 1). By contrast, a clear difference between Sample A and Sample B was observable in the structural information about the second ZDA aggregates and the HC-BR. Specifically, the $R_{\mathrm{g}, \mathrm{ZDA} 2}$ and $R_{\mathrm{g}, \mathrm{BR}}$ values of Sample A were smaller than those of Sample B, and the $N_{\mathrm{ZDA} 2}$ and $N_{\mathrm{BR} \text {, min }}$ values of Sample A were larger than those of Sample B. We could not determine clear differences between the power law exponents of the ZDA aggregates and the HC-BR of Samples A and B, and the physical meaning of the power law exponent is unclear from the present analysis outside of Porod's region $\left(I(q) \sim q^{-4}\right)$ for larger ZDA aggregates.

To determine the correlations between the structural information elucidated from the SAXS and SANS measurements and the mechanical properties of the present rubber materials, we studied the elastic moduli of both of the samples. Figure 6 presents the stressstrain curves of Samples A and B. The stress increases linearly with the strain in the low strain region $($ strain $<0.05)$ and begins to increase more slowly in the high strain region (strain $>0.05$ ), which is typical behavior for rubber. The tensile modulus was calculated in the linear viscoelastic region (strain $<0.05$ ). The calculated moduli of Samples A and B are 48.4 and $22.8 \mathrm{MPa}$, respectively. The modulus of Sample B, which was crosslinked at a higher temperature than Sample A, is lower than that of Sample A, indicating that the crosslinking temperature is a factor controlling the modulus.

As stated above, we did not discover any clear difference in the sizes and the number densities of the first ZDA aggregates of Samples A and $\mathrm{B}$, so it seems that the structures of the second ZDA aggregates, and the HC-BR are related to the difference in the elastic moduli. The second ZDA aggregates in Sample A are smaller in size and larger in number density than in Sample B, suggesting that the ZDA aggregates were crushed into a larger number of smaller aggregates before the crosslinking reaction. This difference arises because Sample A was molded at a lower temperature $\left(170^{\circ} \mathrm{C}\right)$ than Sample B $\left(230^{\circ} \mathrm{C}\right)$, and the crosslinking reaction thus occurred more slowly in Sample A than in Sample B, allowing the ZDA to be crushed into a larger number of smaller aggregates in Sample A. In addition, the distribution of the second aggregates in Sample A looks more homogeneous than that of Sample B, as shown in Figure 2. The homogeneous distribution of small particles may reduce the possibility of stress concentration. A similar tendency was observed in the size and number density of the HC-BR: the size is smaller and the $N_{\mathrm{BR} \text {, min }}$ is larger in Sample A than in Sample B. The second ZDA aggregates most likely affect the formation of the HC-BR. The ZDA aggregates are speculated to act as a kind of core for the BR, resulting in the formation of high crosslink density rubber around the ZDA aggregates. To discuss the correlation between the ZDA aggregates and the $\mathrm{HC}-\mathrm{BR}$ in more detail, we must evaluate the partial scattering function, which can directly reveal correlations between the ZDA aggregates and the HC-BR by using contrast variation SANS. 22

\section{SUMMARY}

In this paper, we investigated the structure of butadiene rubber crosslinked with metal acrylate (ZDA) by using SAXS and SANS, focusing on whether high crosslink density and low crosslink density regions coexisted in the matrix rubber. The SAXS profiles of the rubber materials swollen with toluene were well fitted using the unified approach model and considering only the hierarchical structure of the ZDA aggregates. By contrast, the SANS profiles of the rubber materials swollen with deuterated toluene were not well described by a model that considered only the contribution of the hierarchical structure of ZDA aggregates, suggesting that high crosslink density rubber (HC-BR) must be considered to reproduce the SANS profiles. Then, considering both the contributions from the HC-BR and the ZDA aggregates, we were able to describe the SANS profiles very well, and for the first time successfully clarified the structure of HC-BR through complementary use of SAXS and SANS. Comparing the results of the tensile measurements with the structural information determined using SAXS and SANS revealed that the structures of the HC-BR and the second ZDA aggregates are related to the mechanical properties of the rubber materials. Taking these findings together, the size and the number density of the HC-BR and the second ZDA aggregates are related to the mechanical strength of the rubber materials studied herein. 


\section{ACKNOWLEDGEMENTS}

We thank Dr Yamaguchi of the Japan Atomic Energy Agency for his kind support in the SANS measurements at Japan Research Reactor-3 SANS-J-II (Proposal No. 2010B-A37), Dr Hiroyasu Masunaga of the Japan Synchrotron Radiation Research Institute for his kind support in the SAXS measurements at SPring-8 BL03XU (Proposal No. 2010B7264), Dr Noboru Ohta of the Japan Synchrotron Radiation Research Institute for his kind support in the SAXS measurements at SPring-8 BL40B2 (Proposal No. 2010A1746) and Dr Masugu Sato of the Japan Synchrotron Radiation Research Institute for his kind support in the USAXS measurements at SPring-8 BL19B2 (Proposal No. 2010B1932)

1 Nagata, N., Sato, T., Fujii, T. \& Saito, Y. Structure and mechanical properties of hydrogenated NBR/Zinc di-methacrylate vulcanizates. J. Appl. Polym. Sci. 53, 103-120 (1994).

2 Yuan, X., Zhang, Y., Peng, Z. \& Zhang, Y. In situ preparation of magnesium methacrylate to reinforce NBR. J. Appl. Polym. Sci. 84, 1403-1408 (2002).

3 Wei, Z., Lu, Y., Yan, S., Meng, Y. \& Zhang, L. Dramatic influence of curing temperature on micro-nano structure transform of HNBR filled with zinc dimethacrylate. J. Appl. Polym. Sci. 124, 288-295 (2012).

4 Wei, Z., Lu, Y., Meng, Y. \& Zhang, L. Improved understanding of in-situ polymerization of zinc dimethacrylate: the solid bulk polymerization. Polymer 53, 1409-1417 (2012).

5 Chu, B. J., Wu, D. Q., Lundberg, R. D. \& Macknight, W. J. Small-angle X-ray scattering (SAXS) studies of sulfonated polystyrene ionomers. 1. Anomalous SAXS. Macromolecules 26, 994-999 (1993).

6 Tsujita, Y., Yasuda, M., Takei, M., Kinoshita, T., Takizawa, A. \& Yoshimizu, H. Structure of ionic aggregates of ionomers. 1. Variation in the structure of ionic aggregates with different acid content and degree of neutralization of ethylene and styrene ionomers. Macromolecules 34, 2220-2224 (2001).

7 Al-Hussein, M., Lohmeijer, B. G. G., Schubert, U. S. \& de Jeu, W. H. Melt morphology of polystyrene-poly(ethylene oxide) metallo-supramolecular diblock copolymer. Macromolecules 36, 9281-9284 (2003).

8 Zhou, C. N., Chan, D. C. \& Winey, I. K. Reconciling STEM and X-ray scattering data to determine the nanoscale ionic aggregate morphology in sulfonated polystyrene ionomers. Macromolecules 41, 6134-6140 (2008).
9 Seitz, E. M., Chan, D. C., Opper, L. K., Baughman, W. T., Wagener, B. K. \& Winey, I. K. Nanoscale morphology in precisely sequenced poly(ethylene-co-acrylic acid) zinc ionomers. J. Am. Chem. Soc. 132, 8165-8174 (2010)

10 Luo, H., Klüppel, M. \& Schneider, H. Study of filled SBR elastomers using NMR and mechanical measurements. Macromolecules 37, 8000-8009 (2004).

11 Steren, C. A. \& Monti, G. A. Contribution of the methine group to the transverse ${ }^{1} H$ NMR relaxation in vulcanized natural rubbers. Macromolecules 37, 5624-5629 (2004).

12 Hayashi, S. \& Komori, Y. Determination of residual dipolar interaction from transverse ${ }^{1} \mathrm{H}$ NMR relaxation in elastomers. Solid State Nucl. Magn. Reson. 36, 167-171 (2009).

13 Shiga, K., Murakami, R., Shigemitsu, T., Kimura, H. \& Kitago, T. Golf ball material and golf ball patent appl. no. 207716(JP) (2011).

14 Takenaka, M., Nishituji, S., Amino, N., Ishikawa, Y., Yamaguchi, D. \& Koizumi, S. Structure analyses of swollen rubber-filler systems by using contrast variation SANS. Macromolecules 42, 308-311 (2009).

15 Ikeda, Y., Higashitani, N., Hijikata, K., Kukubo, Y., Morita, Y., Shibayama, M., Osaka, N., Suzuki, T., Endo, H. \& Kohjiya, S. Vulcanization: new focus on a traditional technology by small-angle neutron scattering. Macromolecules 42, 2741-2748 (2009).

16 Suzuki, T., Osaka, N., Endo, H., Shibayama, M., Ikeda, Y., Asai, H., Higashitani, N., Kokubo, Y. \& Kohjiya, S. Nonuniformity in cross-linked natural rubber as revealed by contrast-variation small-angle neutron scattering. Macromolecules 43, 1556-1563 (2010).

17 Russell, T. P., Lin, J. S., Spooner, S. \& Wignall, G. D. Intercalibration of small-angle X-ray and neutron scattering data. J. Appl. Cryst. 21, 629-638 (1988).

18 Kishimoto, $\mathrm{H}$. Shinohara, $\mathrm{Y}$, Amemiya, $\mathrm{Y}$, Inoue, $\mathrm{K}$, Suzuki, $\mathrm{Y}$, Takeuchi, A. Uesugi, K. \& Yagi, N. Structural analysis of filler in rubber composite under stretch with time-resolved two-dimensional ultra-small-angle X-ray scattering. Rubber Chem. Technol. 81, 541-551 (2008).

19 Beaucage, G. Approximations leading to a unified exponential/power-law approach to small-angle scattering. J. Appl. Cryst. 28, 717-728 (1995).

20 Beaucage, G. Small-angle scattering from polymeric mass fractals of arbitrary massfractal dimension. J. Appl. Cryst. 29, 134-146 (1996).

21 Beaucage, G., Kammler, H. K. \& Pratsinis, S. E. Particle size distributions from small-angle scattering using global scattering functions. J. Appl. Cryst. 37, 523-535 (2004).

22 Endo, H., Mihalescu, M., Monkenbusch, M., Allgaier, J., Gompper, G., Richter, D., Jakos, B., Sottmann, T., Strey, R. \& Grillo, I. Effect of amphiphilic block copolymers on the structure and phase behavior of oil-water-surfactant mixtures. J. Chem. Phys. 115, 580-600 (2001). 